\title{
The IncRNA Firre anchors the inactive $X$ chromosome to the nucleolus by binding CTCF and maintains H3K27me3 methylation
}

\author{
Fan Yang ${ }^{1+}$, Xinxian Deng ${ }^{1+}$, Wenxiu Ma ${ }^{2}$, Joel B Berletch ${ }^{1}$, Natalia Rabaia ${ }^{3}$, Gengze Wei ${ }^{4}$, James M Moore ${ }^{3}$,
} Galina N Filippova ${ }^{3}$, Jun Xu4, Yajuan Liu', William S Noble ${ }^{2,5}$, Jay Shendure ${ }^{2}$ and Christine M Disteche $e^{1,6^{*}}$

\begin{abstract}
Background: In mammals, X chromosome genes are present in one copy in males and two in females. To balance the dosage of $X$-linked gene expression between the sexes, one of the $X$ chromosomes in females is silenced. $X$ inactivation is initiated by upregulation of the IncRNA (long non-coding RNA) Xist and recruitment of specific chromatin modifiers. The inactivated $X$ chromosome becomes heterochromatic and visits a specific nuclear compartment adjacent to the nucleolus.

Results: Here, we show a novel role for the IncRNA Firre in anchoring the inactive mouse X chromosome and preserving one of its main epigenetic features, H3K27me3. Similar to Dxz4, Firre is X-linked and expressed from a macrosatellite repeat locus associated with a cluster of CTCF and cohesin binding sites, and is preferentially located adjacent to the nucleolus. CTCF binding present initially in both male and female mouse embryonic stem cells is lost from the active $X$ during development. Knockdown of Firre disrupts perinucleolar targeting and H3K27me3 levels in mouse fibroblasts, demonstrating a role in maintenance of an important epigenetic feature of the inactive $X$ chromosome. No X-linked gene reactivation is seen after Firre knockdown; however, a compensatory increase in the expression of chromatin modifier genes implicated in $X$ silencing is observed. Further experiments in female embryonic stem cells suggest that Firre does not play a role in $X$ inactivation onset.
\end{abstract}

Conclusions: The $X$-linked IncRNA Firre helps to position the inactive $X$ chromosome near the nucleolus and to preserve one of its main epigenetic features.

\section{Background}

Mammalian X chromosome inactivation (XCI) results in random silencing of one of the two $\mathrm{X}$ chromosomes in females in order to balance the dosage of X-linked gene expression between the sexes [1]. XCI is initiated by upregulation of the long non-coding RNA (lncRNA) Xist triggered by the loss of pluripotency factors during early development [2,3]. Xist RNA coats the inactive $\mathrm{X}$ chromosome $(\mathrm{Xi})$ in cis and recruits specific chromatin modifiers for silencing $[4,5]$. Notably, the polycomb repressive complex 2 (PRC2) methylates histone $\mathrm{H} 3$ at

\footnotetext{
* Correspondence: cdistech@u.washington.edu

${ }^{\dagger}$ Equal contributors

'Department of Pathology, University of Washington, Seattle, Washington 98195, USA

${ }^{6}$ Department of Medicine, University of Washington, Seattle, Washington 98195, USA

Full list of author information is available at the end of the article
}

lysine 27 (H3K27me3), leading to chromatin compaction [6]. Other chromatin modifications that accumulate on the inactive $\mathrm{X}$ are H3K9me2-3, H2AK119ub, and H4K20me1. Later, additional epigenetic changes such as DNA methylation at $\mathrm{CpG}$ islands, deposition of macroH2A, and late replication lock in silencing to ensure stability and faithful transmission of the inactive state to daughter cells $[7,8]$. The primary silencing step, that is, Xist expression, was initially thought to be dispensable for the maintenance of XCI [9], but subsequent studies show that Xist, in synergy with epigenetic modifications on the $\mathrm{Xi}$, is needed for long-term persistence of silencing [10]. Interestingly, loss of Xist or of anyone of the histone modifications, for example H3K27me3 or macroH2A, is not sufficient to induce rapid X-linked gene reactivation in somatic cells, confirming that stable 
long-term silencing relies on several layers of epigenetic changes [11-15].

Although most genes on the $\mathrm{Xi}$ are silenced, some genes escape $\mathrm{XCI}$ and remain expressed within the heterochromatic context [16]. In mice, only about $3 \%$ to $6 \%$ of mouse X-linked genes consistently escape XCI based on RNA-sequencing analyses using single nucleotide polymorphisms (SNPs) to distinguish expression from each allele [17-20]. Escape from XCI results in significant sexual dimorphisms in levels of gene expression, suggesting that escape genes may be important for female-specific functions, including XCI. One of the genes found to escape XCI in human and mouse is the IncRNA FIRRE/Firre (previously named 6720401G13Rik in mouse), which represents a macrosatellite repeat located a great distance away from the XIC (X inactivation center) $[17,21]$. Firre has previously been shown to bind hnRNPU and serve as a platform for trans-chromosomal associations involved in the regulation of pluripotency pathways in male embryonic stem (ES) cells [21], but its role in relation to XCI has not been investigated.

The position of the $\mathrm{X}$ chromosomes within the nucleus changes during initiation and maintenance of XCI [22]. Prior to XCI the two active X chromosomes adopt random positions in the nucleus, followed by pairing to ensure correct sensing and counting [23,24]. Once chosen, the Xi forms the heterochromatic Barr body that occupies a discrete and condensed compartment from which active epigenetic marks are excluded $[25,26]$. Furthermore, the Xi frequently visits the perinucleolar region during $\mathrm{S}$ phase, probably for heterochromatin replication and maintenance [27]. Factors important in such positioning are still elusive. One candidate is the 11-zinc finger protein CTCF (CCCTC binding factor). CTCF has been implicated in diverse functions throughout the genome, including control of transcription, promoter/enhancer interactions, chromatin insulation, and nuclear organization [28-30]. This functional diversity depends on which of the 11 CTCF zinc fingers binds to chromatin [31], and on factors that form complexes with CTCF [32]. In concert with nucleophosmin, cohesin, and/or A-type lamins, CTCF tethers chromatin to specific nuclear compartments for gene regulation [33-36]. Interestingly, immunofluorescence analyses show a focal accumulation of CTCF inside the Barr body [26,37], which overlaps with the lncRNA DXZ4 locus known to specifically binds CTCF on the Xi $[38,39]$. Potential interactions between DXZ4 and FIRRE have been reported and $\mathrm{Hi}-\mathrm{C}$ studies further show that the $\mathrm{Xi}$ condenses in two major domains separated by DXZ4 in human cells, suggesting a role in the Xi structure $[39,40]$.

To investigate the role of Firre in mouse XCI we performed allele-specific analyses to demonstrate that this locus specifically binds CTCF and cohesin on the Xi.
The Firre locus on the Xi but not the Xa (active X) was found to be located adjacent to the nucleolus. Knockdown of Firre RNA in mouse fibroblasts disrupted this perinucleolar location and caused a decrease in H3K27me3 enrichment especially on the $\mathrm{X}$ chromosome, whereas knockdown in female ES cells had no effects on XCI onset as determined by Xist RNA accumulation nor on G6pdx gene silencing. These studies reveal a novel role for a lncRNA located away from the XIC in positioning and in maintenance of a specific epigenetic feature of the silenced $\mathrm{X}$ chromosome in somatic cells.

\section{Results}

Firre binds CTCF and cohesin specifically on the inactive $\mathrm{X}$ chromosome

Comparisons of CTCF occupancy profiles between male and female mouse tissues by ChIP-chip identified a striking female-specific CTCF-binding cluster located at the IncRNA Firre locus several $\mathrm{Mb}$ away from the XIC (Figure 1). The Firre locus is approximately $80 \mathrm{~kb}$ in length and contains two subregions, each tandemduplicated and arranged head-to-tail, thus meeting the definition of a macrosatellite repeat (Figure 1A). One set of duplicons $(3.4 \mathrm{~kb})$ has $94 \%$ sequence identity and the other $(6.2 \mathrm{~kb}), 92 \%$ identity. The macrosatellite repeat is GC-rich and enriched in simple repeats, but is remarkably devoid of LINE repeats. RAD21, a component of the cohesin complex, was also bound to Firre only in female mouse tissues (Figure 1B) as expected from the known frequent co-localization of CTCF and cohesin [41-44]. The homologous FIRRE locus in human is also enriched in CTCF, as well as in two cohesin components (RAD21, SMC3) and YY1 specifically in female but not male cells based on occupancy profiles from ENCODE (Figure 1C) [45].

To examine CTCF occupancy on the mouse Xa and $\mathrm{Xi}$ separately, allele-specific ChIP-seq was done using in vitro and in vivo F1 mouse systems in which $\mathrm{XCI}$ is completely skewed and alleles differ by frequent SNPs (1 per 50 to $100 \mathrm{bp)}[17,46,47]$. Allele-specific CTCF occupancy profiles generated using Patski cells (Xi from C57BL/6J) or adult brain (Xi from Mus spretus), showed that CTCF binding was specific to the $\mathrm{Xi}$ allele of the Firre locus (Figure 2A). CTCF motif analysis using FIMO (Find Individual Motif Occurrences) [48], based on a previous study that identified three types of motifs: core (C), upstream (U), and downstream (D) [31], showed that most CTCF motifs within the Firre locus were of the common C-type (68/70 on the BL6 allele and $65 / 67$ on the spretus allele), with only two motifs of the rarer D-type (in the first and third introns). Allelespecific occupancy profiles of RNA polymerase II phosphorylated at serine 5 (PolII-S5p), which is associated with active transcription, showed similarities to the 


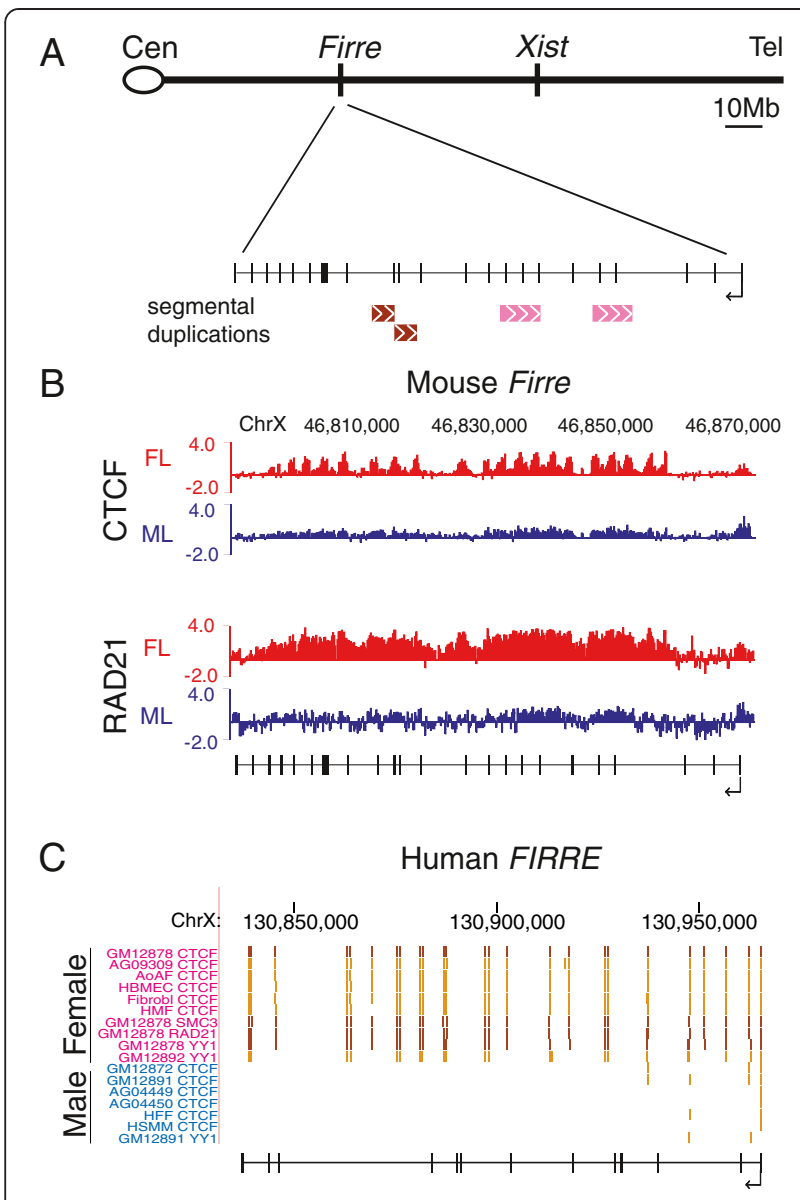

Figure 1 The Firre/FIRRE loci bind CTCF and RAD21 in mouse and human females. (A) Schematic of the mouse $X$ chromosome showing the location of Firre, and Xist. Blow-up shows the IncRNA Firre (transcription direction marked by an arrow) with the location of segmental duplications of tandem repeats (same color indicates paired duplicated regions). Cen, centromere; Tel, telomere. (B) CTCF and RAD21 are bound to Firre in female liver (FL) but not in male liver (ML). ChIP-chip data are shown as $\log _{2}$ ChIP/input. Genomic coordinates are shown at top. (C) CTCF, cohesin (SMC3 and RAD21), and $Y Y 1$ are bound to FIRRE in female (red) but not in male human B-lymphocytes (blue). Peak center tracks (darker color indicates peak strength) from the human ENCODE project [45]. Genomic coordinates are shown at top.

CTCF occupancy profiles, in agreement with the known role of CTCF in transcription (Figure 2B). Note that there were striking differences in the PolII-S5p profiles between the $\mathrm{Xi}$ and $\mathrm{Xa}$ (see below). Next, we tested whether CTCF interacts with Firre RNA itself by reanalysis of published data on allele-specific CTCF-RNA binding in mouse ES cells after 3 days of differentiation [49]. CTCF was found to bind to Firre RNA transcribed from either the $\mathrm{Xa}$ or the $\mathrm{Xi}$, with some differences between alleles (Figure 2C). Thus, CTCF interacts with the Firre genomic locus on the $\mathrm{Xi}$ as well as with the lncRNA itself.
A second cluster of CTCF binding sites on the Xi was observed at $D x z 4$, another macrosatellite repeat associated with a lncRNA, 4933407K13Rik, confirming previous observations [50]. The CTCF binding sites within the mouse Firre and $D x z 4$ loci were found to be highly conserved in human (>90\% identity), suggesting that the CTCF-based functions of these regions are conserved between mammalian species.

\section{Sex-specific changes in CTCF binding and expression of Firre and Dxz4 during development}

To follow CTCF binding at the Firre locus before and after the onset of XCI, ChIP-chip was performed in female (PGK12.1) and male (WD44) mouse ES cells before (day 0) and after differentiation (day 15), as well as in mouse embryos (12.5 dpc) and adult tissue (liver). Surprisingly, the Firre locus was bound by CTCF in both female and male ES cells at day 0, indicating binding to the $\mathrm{Xa}$ in undifferentiated ES cells (Figure 3A). At day 15 after differentiation median CTCF occupancy at the locus increased in female but not in male ES cells (Figure 3B). At later stages of male development (12.5 dpc embryos and adult liver) CTCF binding decreased, whereas it persisted in the corresponding female tissues, consistent with loss of binding on the $\mathrm{Xa}$ and retention on the Xi (Figures 1B, 2A, 3A,B). Accordingly, median CTCF occupancy at the locus was significantly decreased in male embryos (38\% decrease) and liver (30\% decrease) compared to ES cells $(P<2 \mathrm{e}-16$, Wilcoxon Rank-Sum test; Figure $3 \mathrm{~B})$, while there was no significant change between the corresponding stages in females $(P=0.86$ and 0.24 , respectively) (Figure $3 \mathrm{~B}$ ). Similar changes were observed at the Dxz4 locus (Figure 3D). Thus, CTCF binding at the Firre and Dxz4 loci is specifically maintained only on the $\mathrm{Xi}$.

We next examined Firre expression during development. By quantitative RT-PCR (qRT-PCR) the level of Firre RNA was much higher in ES cells than in adult liver (Figure 3C), consistent with expression levels we previously reported in mRNA-seq datasets (14 RPKM (reads per kilobase of exon per million mapped reads) in PGK12.1 and 0.9 RPKM in mouse liver) [47]. Note that the fold change detected by qRT-PCR analysis was greater than that detected by RNA-seq, probably because the latter only detects mature RNA with polyA tails, while the RT-PCR amplicon may target alternative and/or short transcripts from the macrosatellite repeat locus (Additional file 1: Figure S1). As expected given that Firre escapes XCI $[17,21]$, expression was significantly higher in females not only in undifferentiated ES cells prior to XCI but also in differentiated ES cells and in liver, compared to the corresponding male tissues (Figure 3C). Firre expression from both the $\mathrm{Xi}$ and the $\mathrm{Xa}$ was supported by PolII-S5p occupancy on both alleles: 10 to 12 peaks of 

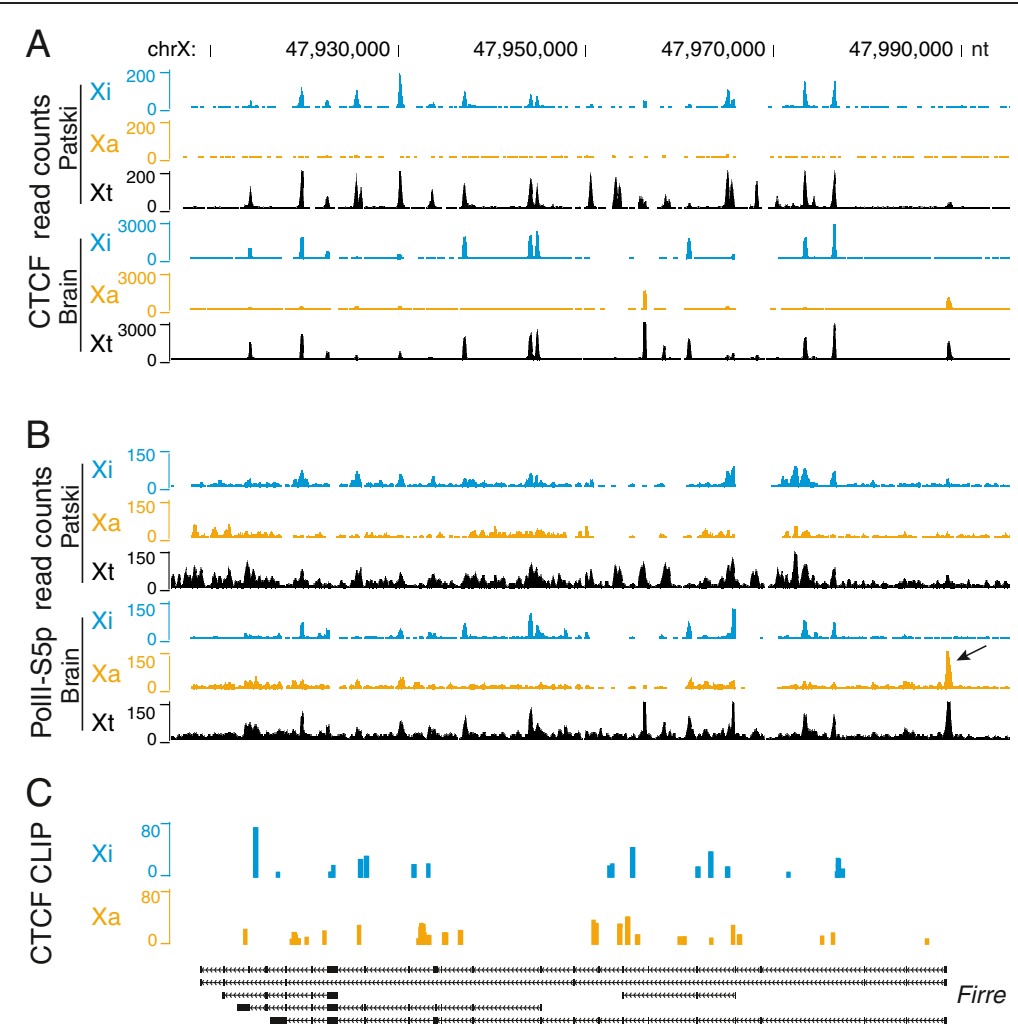

Figure $\mathbf{2}$ CTCF and RNA polymerase II are present at the Firre locus on the Xi allele and CTCF interacts with the IncRNA. Allele-specific CTCF (A) and Polll-S5P (B) profiles were established based on SNP analyses of ChIP-seq results in Patski cells and in F1 mouse brain, both derived from crosses between C57BL/6 J and M. spretus. In Patski cells the Xi is from BL6 while in the brain, the Xi is from spretus X. Allele-specific sequence read counts for the Xi (blue) and the Xa (orange), and total mapped read counts (Xt) are shown in 100 bp windows. A few peaks visible in the Xt track could not be assigned to an allele due to a lack of informative SNPs. Polll-S5p peaks are present in multiple regions of Firre on the Xi, indicating alternative transcript start sites. The arrow points to a Polll-S5p peak present only on the Xa at the very 5'end of the Firre transcript in brain. Genomic coordinates are shown at the top and the position of alternative Firre transcripts, at the bottom (from UCSC). (C) CTCF-RNA interactions obtained by CLIP-seq based on re-analysis of published data [49]. Allele-specific profiles for the Xi (blue) and Xa (orange) show differential patterns of interactions between CTCF and the IncRNA transcribed from each allele.

high PolII-S5p occupancy were dispersed throughout the locus on the $\mathrm{Xi}$, while a single peak was located at $5^{\prime}$ end on the Xa (Figure 2B). Based on these striking differences we conclude that Firre transcripts differ between the Xi and $\mathrm{Xa}$, with multiple alternative start sites on the $\mathrm{Xi}$, possibly generating small transcripts. The lncRNA 4933407K13Rik transcribed at the $D x z 4$ locus was also more highly expressed in female than male cells and liver (Figure 3E). Interestingly, different size transcripts are also generated from each DXZ4 allele in human cells, with short transcripts specifically originating from the $\mathrm{Xi}$ [51].

We performed RNA-FISH for Firre in Patski cells to determine the IncRNA location. Surprisingly, no Firre RNA signal was detected from the Xi marked by the Xist RNA cloud and only one bright signal presumably from the Xa was observed (Additional file 2: Figure S2A). This was confirmed using RNA-FISH in female MEFs (mouse embryonic fibroblasts) and in primary neuronal cells derived from the mouse hippocampus (Additional file 3: Figure S3). The failure to detect a Firre RNA signal on the Xi even though RNA-seq and PolII occupancy profiles clearly indicate transcription from the Xi could be due to low Xi-expression that represents only $16 \%$ of Xa-expression based on allelic RNA-seq (Additional file 4: Table S1), and/or to low sensitivity of RNA-FISH for the detection of short alternative transcripts on the Xi. Likewise, a previous study reported that RNA-FISH failed to detect a signal on the $\mathrm{Xi}$ in mouse lung fibroblasts unless Firre was ectopically overexpressed many folds [21].

\section{Firre and $D x z 4$ alleles on the inactive $X$ chromosome associate with the nucleolus}

The location of the Firre and Dxz4 loci within the nucleus was determined by DNA-FISH using labeled BAC clones (RP23-338M16 for Firre and RP23-299L1 for $D x z 4)$ in combination with immunofluorescence with an anti-nucleophosmin antibody. At least 100 nuclei were scored for each experiment to evaluate the association between the loci and the nucleolus, as defined by the adjacent location of one FISH signal to the nucleolus 


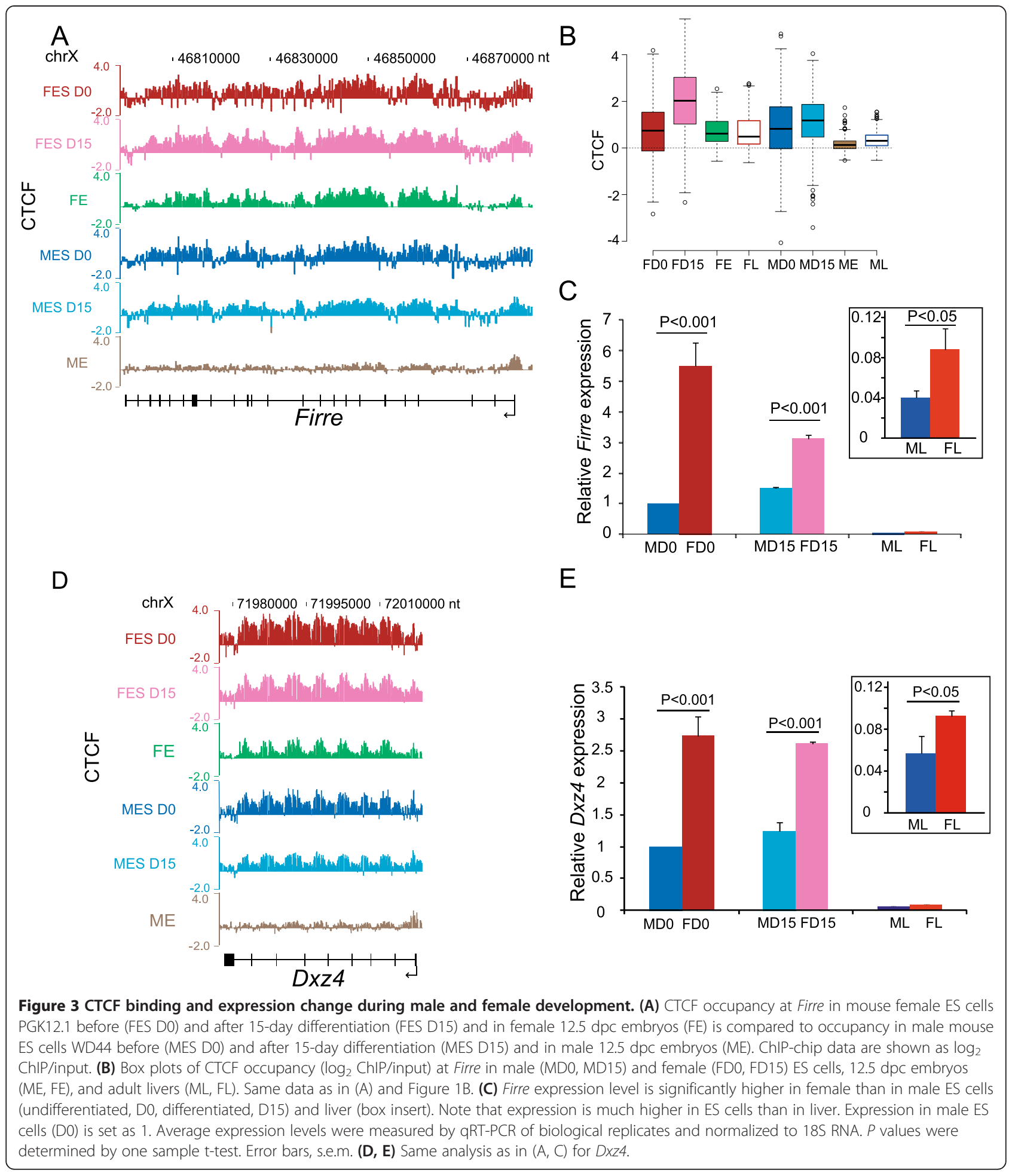

surface (Additional file 5: Figure S4). Firre association to the nucleolus was much more frequent in female compared to male cells (Figure 4A; Additional file 5: Figure S4A-C). Indeed, we observed association in $58 \%$ of female fibroblasts (Patski), but only in 17\% of male ear fibroblasts
(Figure 4B). Since female cells have two X chromosomes the predicted percentage of nuclei with background nucleolar association for at least one signal was estimated to be $34 \%$ based on observations in male nuclei. Accordingly, a control BAC clone (RP23-112D6) for a region not 

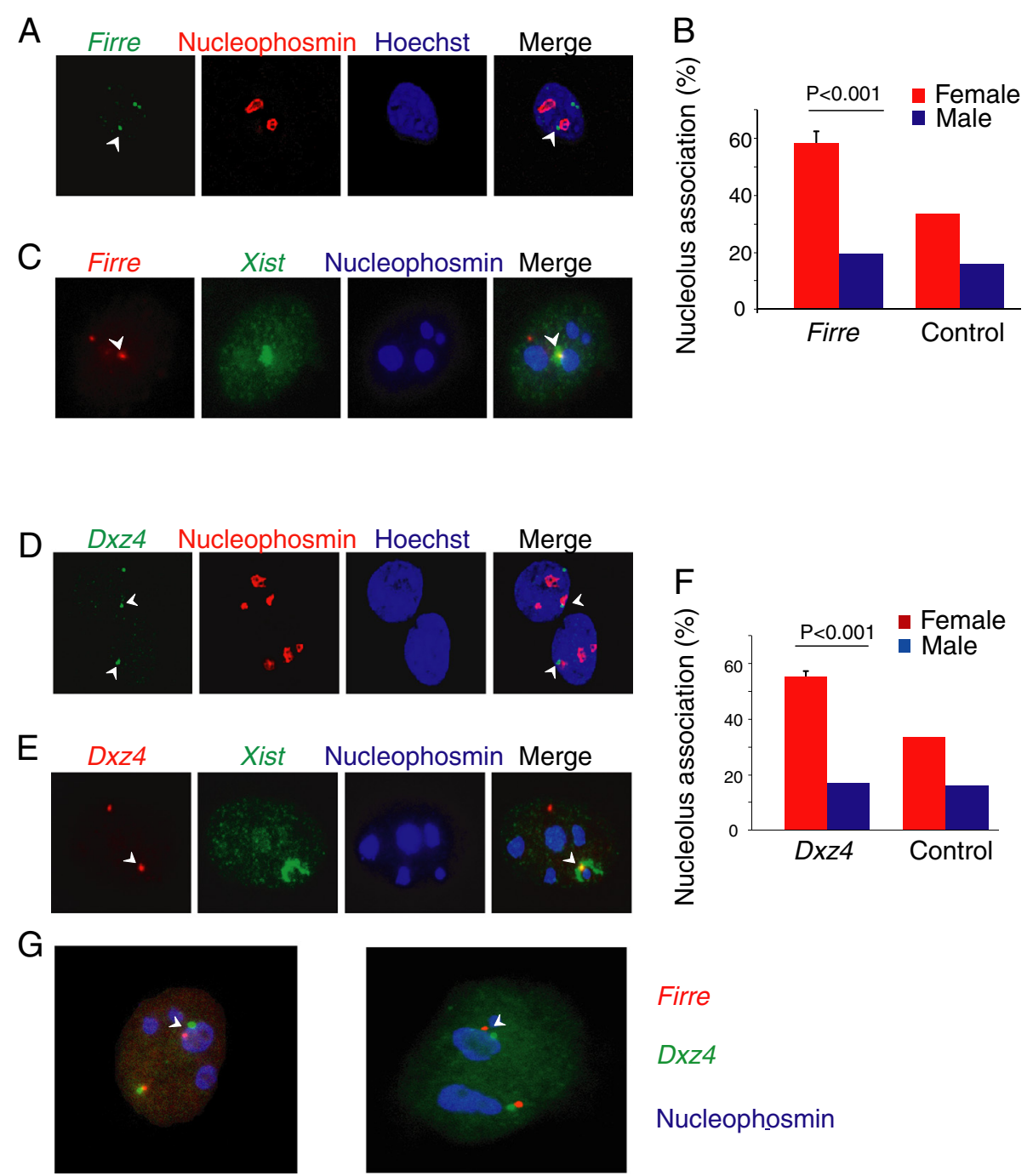

Figure 4 Firre and Dxz4 on the Xi are associated with the nucleolus in female nuclei. (A) Examples of nuclei from Patski cells after DNA-FISH to detect Firre (green signals), immunostaining to detect nucleophosmin (red) on the surface of the nucleolus, and counterstaining with Hoechst 33342 (blue). Firre association with the nucleolus is marked by an arrowhead. One deconvoluted Z section is shown. (B) Frequency of association (in \% of nuclei) between the nucleolus and Firre or a control BAC clone (RP23-112D6) in female Patski cells (red) and male ear fibroblasts (blue). $P$ values were determined by one sample t-test. Error bars, s.e.m. (C) Examples of nuclei from Patski cells after DNA-FISH to detect Firre (red), RNA-FISH to detect Xist (green) and mark the Xi, and immunostaining to detect nucleophosmin (blue). One deconvoluted Z section is shown. (D-F) Same analysis as in A-C for Dxz4 (see also Additional file 5: Figure S4D). (G) Firre and Dxz4 on the Xi are separately associated with the nucleolus in female nuclei. Example of nuclei from Patski cells after DNA-FISH to detect Firre (red), Dxz4 (green), and immunostaining to detect nucleophosmin (blue) on the surface of the nucleolus (see also Additional file 5: Figure S4A).

enriched in CTCF binding showed background levels of nucleolar association in $34 \%$ and $16 \%$ of female and male fibroblasts, respectively (Figure 4B). The increased association of the Firre locus with the nucleolus surface in female versus male cells was highly significant $(P=0.0008$ by one-sample t-test). To identify the Firre allele that associated with the nucleolus surface the Xi domain was marked by RNA-FISH using an Xist probe labeled a different color. A clear overlap between the Xist RNA cloud and the Firre DNA signal located adjacent to the nucleolus identified the $\mathrm{Xi}$ allele as the one targeted to the perinucleolar region (Figure 4C). Similar results were obtained for Dxz4 (Figure 4D-F; Additional file 5: Figure S4D,E). However, DNA-FISH signals obtained using different color probes for the two loci did not overlap, suggesting that the Firre and Dxz4 loci did not aggregate within a single complex (Figure 4G, Additional file 5: Figure S4A). Based on these results, we conclude that there is independent association between the two CTCFbinding clusters on the $\mathrm{Xi}$ and the nucleolus surface. 


\section{Knockdown of Firre RNA decreases perinucleolar localization of the $\mathrm{Xi}$ in fibroblasts}

We next examined the effects of Firre RNA depletion. Knockdown of Firre using a double siRNA (small interfering RNA) treatment in Patski cells achieved a $>70 \%$ reduction in levels of the lncRNA and resulted in a significantly lower frequency of association between Firre and the nucleolus (from $56 \%$ to $40 \%$; $P=0.0002$ by twotail unpaired student t-test; Figure $5 \mathrm{~A})$. The frequency of $D x z 4$ association to the nucleolus was also reduced (from $58 \%$ to $42 \%, P=0.005$ by one-sample t-test), suggesting that the perinucleolar location of the whole Xi was disrupted (Figure 5A). Since no changes in CTCF and RAD21 occupancy were apparent at the Firre (or Dxz4) locus after knockdown, Firre lncRNA levels apparently influence perinucleolar targeting of the $\mathrm{Xi}$ independent of CTCF and RAD21 binding at the locus (Figure 5B). We also performed Ctcf knockdown and verified depletion by qRTPCR and western blots (Additional file 6: Figure S5A). Knockdown of Ctcf also significantly reduced perinu-

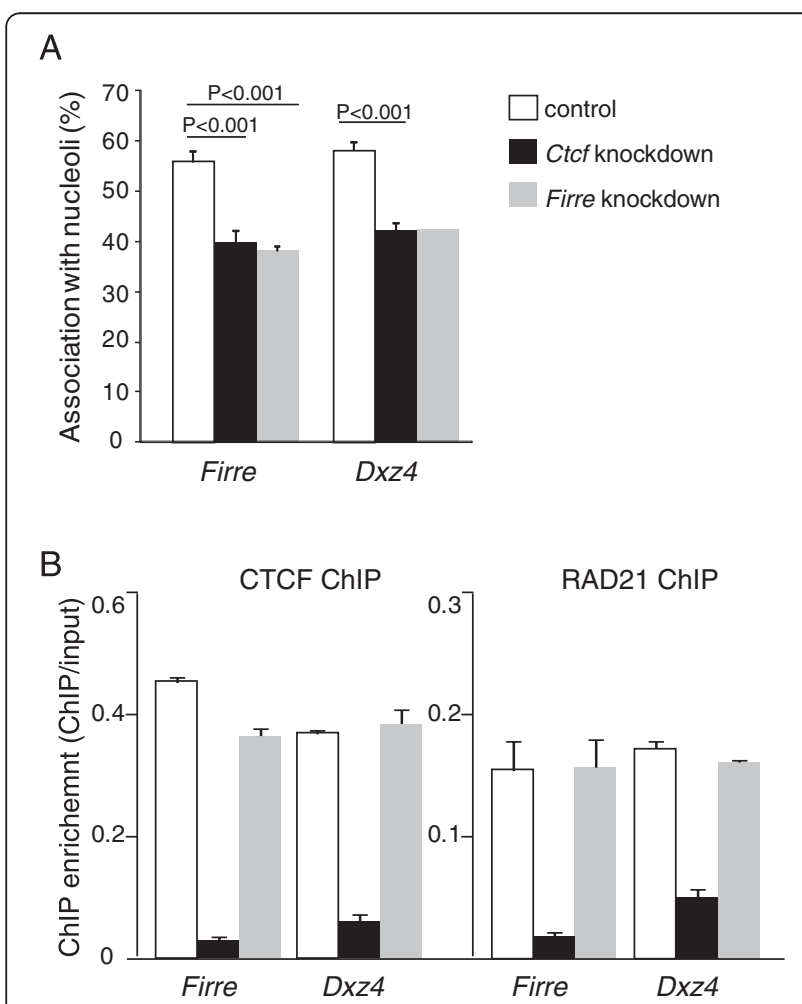

Figure $\mathbf{5}$ Knockdown of Firre or Ctcf decreases association of Firre and Dxz4 to the nucleolus. (A) Association frequency (in \% of nuclei) between Firre or Dxz4 and the nucleolus as determined by DNA-FISH and immunostaining is significantly reduced after either Firre or Ctcf knockdown in Patski cells. At least 100 nuclei were scored in each experiment. $P$ values were determined by two-tail unpaired student t-test. Error bars represent s.e.m. (B) Occupancy by CTCF and RAD21 at Firre and Dxz4 before and after knockdowns of Firre or Ctcf. ChIP enrichment is shown as the ratio between ChIP and input fractions as measured by qRT-PCR. Error bars represent s.e.m. cleolar targeting of both Firre (from 56\% to 39\%, $P=0.0009$ ) and $D x z 4$ (from $58 \%$ to $42 \%, P=0.0004$ ), implying that CTCF was necessary for targeting of the $\mathrm{Xi}$ (Figure 5A). This effect could be indirect since Ctcf knockdown caused a significant reduction in expression of Firre (but not of 4933407K13Rik, data not shown). Furthermore, Xist expression was also decreased (see below), consistent with a previous report in differentiated mouse ES cells [52], and possibly causing a loss in nucleolus association of the $\mathrm{Xi}$ [27]. In contrast, no Xist reduction was observed after Firre knockdown, suggesting that Firre acts independently from Xist. There was a remarkable loss of RAD21 at Firre (and at Dxz4) after Ctcf knockdown, indicating that cohesin binding at these loci is dependent on CTCF (Figure 5B). Taken together, our results suggest that the lncRNA Firre and CTCF occupancy at its genomic locus may help anchoring the Xi to the nucleolus.

\section{Firre and Ctcf knockdowns in fibroblasts cause a loss of H3K27me3 on the Xi}

To determine whether Firre influences epigenetic features of the Xi H3K27me3 enrichment was compared before and after RNA knockdown. Independent Firre RNA knockdowns were done using either a double siRNA treatment or a combination of shRNA and siRNA in Patski cells. Immunostaining of H3K27me3 in interphase nuclei showed a significant reduction by $76 \%$ in the number of nuclei with an intense spot corresponding to the Xi after Firre knockdown, consistent with a substantial loss of H3K27me3 normally detected on the $\mathrm{Xi}$ (Figure 6A). RNA-FISH showed no significant changes in the frequency or shape of Xist RNA clouds visible in $89 \%$ of Firre knockdown cells compared to $87 \%$ of control cells (Additional file 2: Figure S2B). This, together with no change in Xist RNA levels upon Firre knockdown, strongly suggests that Firre regulates $\mathrm{Xi}$ nucleolus association and H3K27me3 enrichment independent of Xist. The loss of H3K27me3 on the Xi was further demonstrated by metagene analyses based on ChIP-chip for H3K27me3 after two independent Firre knockdowns, either transient (siRNA) or stable (shRNA and siRNA) (Figure 6B-D; Additional file 6: Figure S5B). As expected based on the known high levels of H3K27me3 on the $\mathrm{Xi}$, average enrichment between $+1 \mathrm{~kb}$ and $+2 \mathrm{~kb}$ of the TSS was higher for X-linked genes than for autosomal genes. Importantly, upon Firre knockdown, a clear shift to lower H3К27me3 values was specifically observed for $\mathrm{X}$-linked genes whose average enrichment significantly decreased by $15 \%$ to $17 \%$ ( $P=1 \mathrm{e}-10$, Kolmogorov-Smirnov test) (Figure 6C). Comparisons between X-linked genes showed a greater decrease in H3K27me3 for genes subject to XCI, while escape genes [17] had low H3K27me3 levels to begin with, and little to no change after knockdown (Figure 6D; Additional file 7: Table S2). Note that, despite 
A
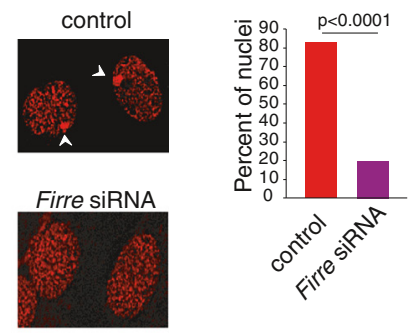

C

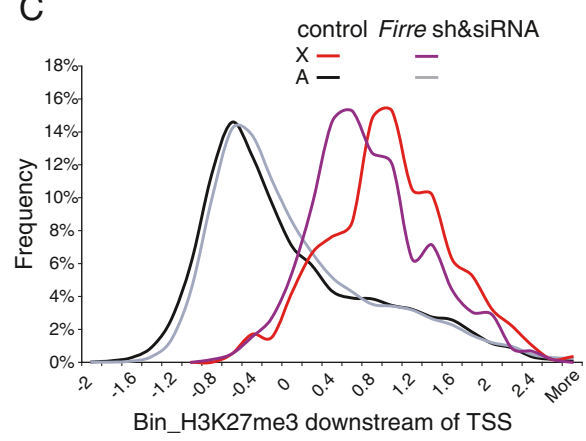

$\mathrm{E}$

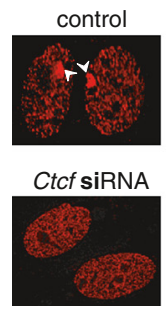

B

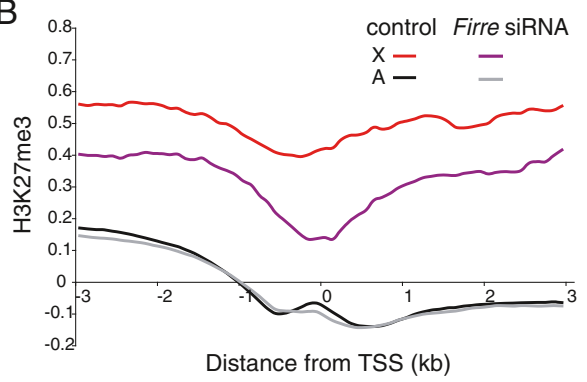

$\mathrm{D}$

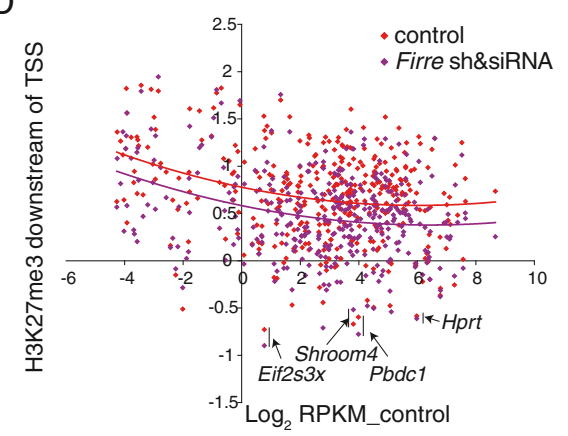

$\mathrm{F}$

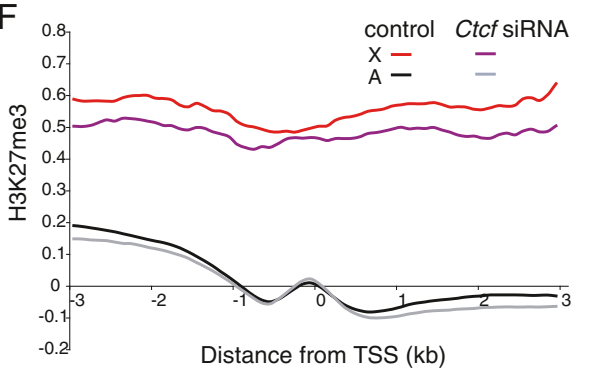

Figure 6 Knockdown of Firre or Ctcf affects H3K27me3 enrichment of the Xi. (A) Examples of interphase nuclei after H3K27me3 immunostaining (left panel) and graph of percent of nuclei with an H3K27me3 intense staining spot (right panel) in control and Firre knockdown Patski cells. The H3K27me3 spot over the Xi (marked by arrowheads) is no longer visible after knockdown. P value was determined by Chi-square tests. (B) H3K27me3 enrichment is reduced at the $5^{\prime}$ end of X-linked genes but not at autosomal genes after Firre knockdown using siRNAs in Patski cells. Metagene analysis shows average H3K27me3 enrichment 3 kb upstream and downstream of the transcription start site (TSS) for 647 X-linked genes $(X)$ versus 16,141 autosomal genes (A). (C) The distribution of average H3K27me3 enrichment at 1 to $2 \mathrm{~kb}$ downstream of the TSS is clearly shifted left to lower values for X-linked genes but not for autosomal genes after Firre knockdown. (D) Scatter plot of H3K27me3 enrichment at 1 to 2 kb downstream of the TSS shows a shift to lower values for most X-linked genes after Firre knockdown (purple) compared to control (red), as indicated by trend lines. Three escape genes, Eif2s3X, Shroom4, and Pbdc1 that show no H3K27me3 enrichment are labeled. Hprt, a gene subject to XCl, shows no H3K27me3 enrichment because it is deleted in Patski cells. (E, F) Same analysis as in (A, B) but for Ctcf knockdown. There is a lesser decrease in H3K27me3 levels on the X than in Firre knockdown.

being subject to XCI, Hprt had no apparent H3K27me3 enrichment, due to its deletion from the $\mathrm{Xi}$ in Patski cells (Figure 6D) [46].

After Ctcf knockdown a lesser effect on the percentage of nuclei with an intense spot of H3K27me3 (71\% decrease) and on the average $\mathrm{X}$-specific enrichment measured by metagene analysis ( $5 \%$ decrease) were observed (Figure 6E, F). We conclude that Firre and to a lesser extent CTCF are important for maintenance of H3K27me3 on the $\mathrm{Xi}$.

\section{Gene expression analyses after Firre knockdown in fibroblasts}

RNA-seq was initially used to compare gene expression in Patski cells between control and Firre knockdown obtained using a combination of shRNA and siRNA (see Methods for details; Additional file 4: Table S1). For both expressed ( $\geq 1$ RPKM) X-linked genes (342) and autosomal genes $(11,182)$ the median fold changes were about 1.0 ( 0 in $\log _{2}$ scale; Figure 7A), indicating no overall change in gene expression after knockdown. However, 


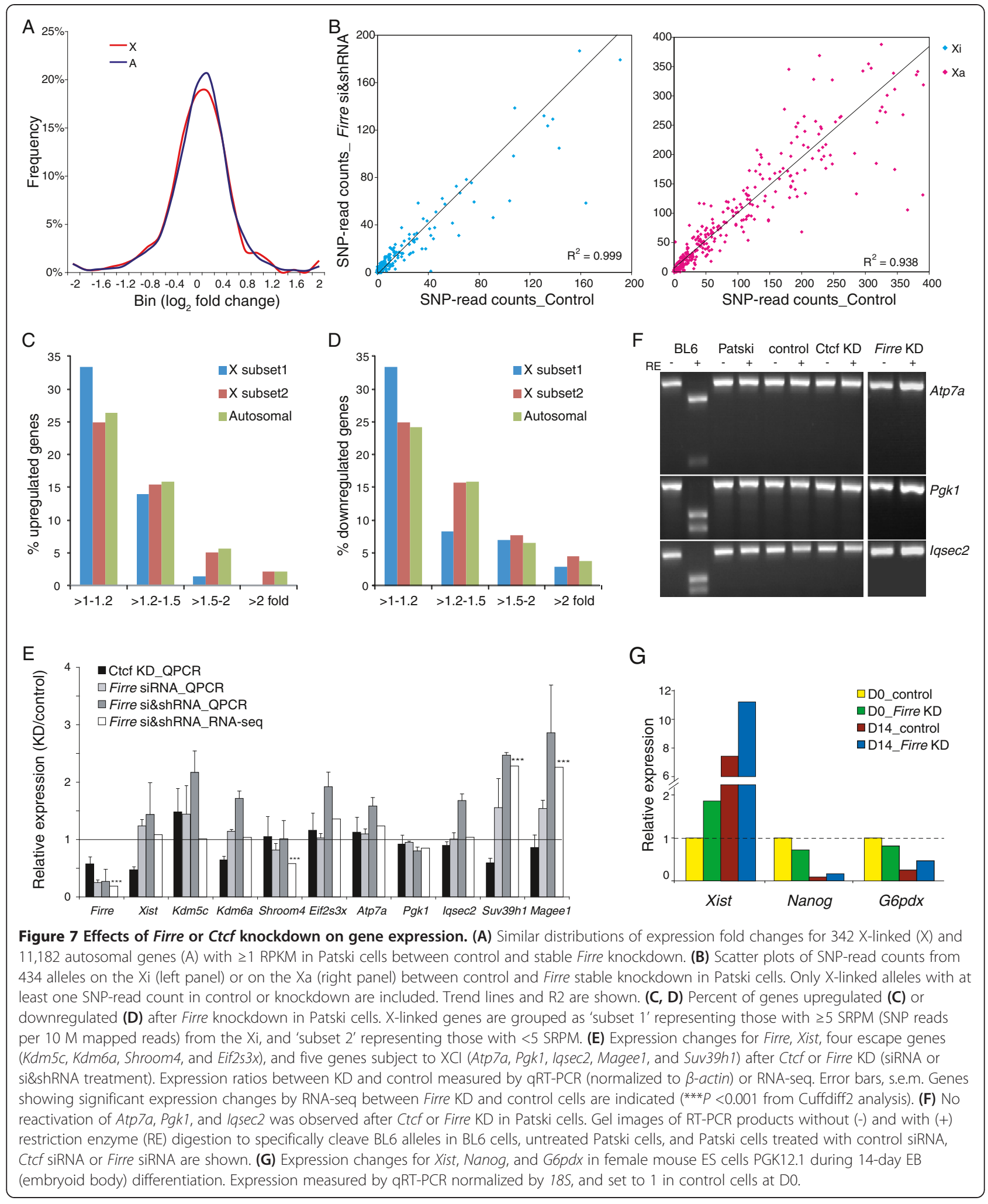

subsets of genes representing 33 (10\%) X-linked and $1,218(11 \%)$ autosomal genes were significantly downregulated, while 17 (5\%) X-linked and 811 (7\%) autosomal genes were significantly upregulated $(>1.25$-fold expression change and $P<0.05$ from Cuffdiff2 analysis; Additional file 4: Table S1). Similar trends were observed when using 1.5- or 2.0-fold cutoffs. Upregulated genes (but not downregulated genes) were enriched in 
distinct functional categories such as nucleus, DNAbinding, chromosomal proteins, and chromatin regulating proteins, based on analysis by GO DAVID [53] (Additional file 8: Table S3). Interestingly, a subset of these upregulated genes encode protein components of the PRC1 complex (Bmi1, Cbx6, and Rnf2) and proteins implicated in heterochromatin regulation (Cbx1, Suv39h1) or in maintenance of DNA methylation (Dnmt1). These proteins are known to mediate the deposition of $\mathrm{X}$ silencing marks such as H3K9me2-3, H2AK119ub, H2b, and DNA methylation [13] (Additional file 9: Table S4). In contrast, Suz12, a gene that encodes a core subunit of the PRC2 complex that controls H3K27me3, had a 30\% decrease in expression, consistent with loss of H3K27me3 on the Xi. No expression changes were detected at genes that encode CTCF or hnRNPU (data not shown), which are involved in Xist regulation/coating [54,55]. We next examined allelespecific X-linked gene expression in Patski cells and found little evidence of reactivation from the Xi alleles after Firre knockdown (Figure 7B; Additional file 10: Table S5). When considering a subset of X-linked genes with $\geq 5$ SNP-reads per 10 million mapped reads from the allele on the $\mathrm{Xi}$ (in control, Firre knockdown, or both) only 1/73 (1\%) of these escape genes showed evidence of $>1.5$ fold upregulation, compared to other X-linked genes (23/265 or 9\%) or to autosomal genes (854/11039 or 7\%) (Figure 7C,D; Additional file 11: Table S6). This reflects lower levels of H3K27me3 on the Xi at escape genes (see above), resulting in a lesser impact of Firre knockdown compared to genes subject to XCI (Figure 6D; Additional file 7: Table S2). Taken together, our findings suggest that the lack of significant X-linked gene reactivation following depletion of Firre may be due to a compensatory increase in expression of genes that maintain $\mathrm{X}$ silencing. Such compensation reflects the multiple layers of regulation that control XCI $[12,14,53]$.

Allele-specific expression analyses of individual genes using qRT-PCR and RT-PCR combined with restriction endonuclease digestion to selectively cleave the BL6 allele on the $\mathrm{Xi}$ were in general agreement with RNA-seq results. Atp7a, Pgk1, and Iqsec2 representing genes normally subject to XCI did not show any significant expression changes after either Firre or Ctcf knockdown (Figure 7E,F). In contrast, two other genes normally subject to XCI, Suv39h1, and Magee1, were significantly upregulated, but this increased expression was from the Xa allele with no reactivation of the $\mathrm{Xi}$ allele (Figure 7E; Additional file 4: Table S1). Three genes that escape $\mathrm{XCI}, K d m 5 c, K d m 6 a$, and Eif2s3x, showed no consistent expression changes by RT-PCR (Figure 7E; Additional file 12: Table S7). Again, Xist expression did not decrease but rather increased by approximately 1.5 -fold after Firre knockdown, consistent with RNA-seq results (Figure 7E; Additional file 12: Table S7). Note that expression of Firre was significantly decreased (1.7-fold; $P=0.0008$ by one-sample t-test) after Ctcf knockdown, suggesting that CTCF regulates expression of the lncRNA (Figure 7E).

\section{Gene expression analyses after Firre knockdown in female ES cells}

To investigate the role of Firre in XCI initiation, we obtained a stable Firre knockdown using shRNA in female mouse ES cells PGK12.1 (Additional file 13: Figure S6A). Unlike what was reported for a complete Firre knockout, which causes a marked retardation in ES cell growth rate and colony formation [21], we did not observe dramatic growth differences between control and knockdown cells (data not shown). In addition, the master pluripotency gene Nanog was upregulated approximately 10-fold following differentiation, consistent with normal differentiation in knockdown PGK12.1 cells (Figure 7G). Both Firre knockdown and control ES cells had more than an eight-fold increase in Xist at day 14 compared to day 0, indicating that the knockdown did not affect Xist upregulation (Figure 7G). In fact, there was a 1.5- to 2-fold increase in Xist expression in Firre knockdown versus control ES cells regardless of their differentiation status, similar to what we observed in Patski cells (Additional file 13: Figure S6B). Xist RNA-FISH showed no changes in the shape of the Xist cloud between control and Firre knockdown cells at day 14 (Additional file 13: Figure S6C). We next used RT-PCR to measure the expression of $G 6 p d x$, a gene known to display a two-fold decrease in expression due to XCI upon differentiation of female ES cells [56]. G6pdx expression was decreased about two-fold in control and Firre knockdown cells at day 14 (Figure 7G), indicating that X-linked gene silencing was not disrupted after Firre knockdown in female ES cells. These results suggest that Firre does not play a major role in XCI initiation.

\section{Discussion}

Here we have shown that the lncRNA Firre locus is involved in two interrelated features of the inactive $\mathrm{X}$ chromosome in terms of positioning near the nucleolus and maintenance of H3K27me3.

\section{Firre in nucleolar association of the $\mathrm{Xi}$ via CTCF}

The $\mathrm{Xi}$ often visits the perinucleolar region during $\mathrm{S}$ phase in female mouse embryonic fibroblasts (MEFs) [27]. The dynamic association of the $\mathrm{Xi}$ to the nucleolus is cell-cycle dependent and involves the XIC, visible as a DNA-FISH signal adjacent to the edge of the nucleolus in $50 \%$ to $60 \%$ of cells, a percentage similar to what we observed for Firre and another lncRNA locus Dxz4. We postulate that the macrosatellite loci Firre and Dxz4 located $54 \mathrm{Mb}$ and $29 \mathrm{Mb}$ away from the XIC, 
respectively, may provide additional attachment points to anchor the $\mathrm{Xi}$ via a CTCF/cohesin complex. We cannot exclude that Firre and Dxz4 may also help anchor the $\mathrm{Xi}$ near the nuclear membrane where the $\mathrm{Xi}$ is also frequently found. Both the nuclear membrane and the nucleolus have been identified as preferred 'velcro' sites for heterochromatin [57]. The human IncRNA FIRRE is also bound by CTCF and RAD21 on the Xi, suggesting a conserved role between species [21,39]. Interestingly, perinucleolar targeting and H3K27me3 enrichment on the $\mathrm{Xi}$ are conserved in marsupials even though other mechanistic aspects of $\mathrm{X}$ inactivation differ and Xist is replaced by another IncRNA $R s x[58,59]$.

Components of the cohesin complex, RAD21 and SMC3, co-localize with CTCF at the Firre and Dxz4 loci on the mouse $\mathrm{Xi}$, which may help sequestration of the $\mathrm{Xi}$ to a specific nuclear compartment. Many but not all CTCF sites are known to attract cohesin, likely to organize chromatin within the nucleus [41-44]. Cohesin assists CTCF in targeting genomic DNA or genes to specific subnuclear locations for correct expression [60]. Furthermore, there is prior evidence that CTCF tethers chromatin to the nucleolus via a complex with nucleophosmin [33]. Thus, perinucleolar targeting of the Xi may depend on a multi-protein complex that includes CTCF, cohesin, YY1, and nucleophosmin. In addition, previous observations demonstrate that CTCF accumulation at DXZ4 coincides with the center of the condensed Barr body in human cells, suggesting a role in Xi structure [26,37]. Recent $\mathrm{Hi}-\mathrm{C}$ studies have further shown that two major domains of condensation on the human $\mathrm{Xi}$ are separated by the DXZ4 region [40]. The parallel effects of Ctcf knockdown that we observed at Firre and Dxz4 in terms of disruption of nucleolar targeting suggest that both regions may help anchor the Xi. Interestingly, CTCF helps locate another macrosatellite repeat $D 4 Z 4$ not to the perinucleolar region, but to a compartment adjacent to the nuclear membrane via a complex with A-type lamins [35]. In Drosophila, a protein complex containing CTCF, nucleophosmin and another nucleolar protein Modulo helps anchor centromeric chromatin to the nucleolus. Disruption of components of the complex results in abnormal spatial arrangement of the centromeric regions and in defects of heterochromatin stability and silencing [61]. Thus, while the ubiquitous protein CTCF is clearly an important factor for the correct placement of loci within the nucleus to maintain their chromatin state, this role is implemented through interactions with various other proteins (for example, cohesin, nucleophosmin, A-type lamins) that specify the subnuclear compartment. Specificity of the regions targeted to a site within the nucleus may also depend on motifs at the CTCF binding sites and on the presence of associated lncRNAs. Our re- analyses of previous data [49] are consistent with CTCF binding to Firre lncRNA.

The loss of CTCF binding that we observed specifically on the Xa during male and female ES cells differentiation may be mediated by the onset of DNA methylation that would preclude CTCF from binding. This is supported by our re-examination of published genome-wide profiles obtained by bisulfite sequencing that shows heavy DNA methylation at Firre in male mouse brain [62]. A similar mechanism has been proposed in the context of the enhancer-blocking function of CTCF at the H19/Igf2 locus where DNA methylation at the imprinting control region (ICR) eliminates CTCF binding $[63,64]$. In human, the DXZ4 locus is also heavily methylated on the Xa but completely unmethylated on the Xi $[38,65]$; however, in vitro DNA methylation of the CTCF binding sites apparently does not block CTCF binding, suggesting that the interactions between CTCF and DNA methylation may be more complex. Furthermore, there may be epigenetic differences between species. For example, unlike what has been reported for DXZ4 we did not observe enrichment in H3K9me3 at the corresponding mouse locus [38] (data not shown). Furthermore, we did not detect overlap of the $D x z 4$ and Firre loci, which differs from a previous study indicating aggregation of FIRRE and DXZ4 via CTCF in human cells $[39,40]$.

\section{Firre in maintenance of $\mathrm{H} 3 \mathrm{~K} 27 \mathrm{me} 3$ on the $\mathrm{Xi}$}

Studies in yeast have proposed that lncRNAs may function as assembly platforms for the recruitment of proteins such as histone methyltransferases to heterochromatin nucleation sites [66]. Our findings support a role for the Firre lncRNA in preserving one of the epigenetic characteristics associated with XCI, probably via specific proteins that control H3K27me3. Indeed, expression of Suz12, a gene that encodes a core component of the PRC2 complex for the deposition of H3K27me3 on the Xi was decreased after Firre RNA knockdown. Additional studies will be required to fully understand the molecular mechanisms that govern epigenetic features of the $\mathrm{Xi}$ in relation to nuclear elements such as the nucleolus or the nuclear membrane. A similar type of anchoring mechanism for the regulation of a specific epigenetic feature has been proposed for the lncRNA Kcnq1ot1 that contacts the nucleolus possibly via CTCF and controls H3K27me3 levels at the imprinted locus Kcnq1 [67]. However, perinucleolar localization of Kcnq1ot1 may not be sufficient to preclude transcription of genes in the imprinted domain [68]. Similarly, we did not observe reactivation of $\mathrm{X}$-linked genes upon Firre knockdown, consistent with H3K27me3 representing only one layer of XCI control. A new study supports a role for Firre in nuclear organization via interactions with the nuclear matrix factor hnRNPU to help co-localize genomic 
loci involved in adipogenesis by cis- and trans-interactions [21]. Interestingly, hnRNPU is required for Xist association with the Xi and deposition of H3K27me3 [55].

A similar number of autosomal and X-linked genes were upregulated or downregulated, after Firre knockdown in fibroblasts probably due to indirect effects of disrupting $\mathrm{X}$ heterochromatin and to loss of H3K27me3. Importantly, our results are consistent with Firre having a role in maintenance of H3K27me3 independent of Xist that was not decreased by Firre knockdown. The lack of $\mathrm{X}$-linked gene reactivation we observed following knockdown of either Firre or Ctcf is not surprising given that silencing of the $\mathrm{Xi}$ is extremely stable in somatic cells even after depletion of Xist or of PRC2 [9,15,69,70]. Even removal of DNA methylation by 5 -aza-cytidine does not result in substantial X-linked gene reactivation in somatic cells [71]. Thus, Xist coating, DNA methylation, and histone modifications all contribute in synergism to maintain $\mathrm{X}$ silencing [11]. Interestingly, among those with increased expression upon Firre knockdown were genes that encode components of the PRC1 complex (Bmi1, Cbx6, and Rnf2) and other proteins that also control heterochromatin (Suv39h1, Cbx1) and deposit silencing marks on the Xi [6,13], as well as Dnmt1 implicated in maintenance of DNA methylation, suggesting compensatory silencing mechanisms in response to the loss of H3K27me3. Our analyses were done using short-term knockdown and would not detect long-term effects on gene expression. It is possible that failure to maintain perinucleolar anchoring of the Xi would eventually affect gene expression after multiple cell divisions. Such a delayed effect was demonstrated in the case of Xist whose continued expression appeared dispensable for maintenance of XCI in short-term analyses of cell lines [9], while being essential for long-term maintenance of XCI [10]. Our findings of dramatic differences in CTCF binding between males and females in differentiated ES cells and adult tissues but not in undifferentiated ES cells are consistent with Firre regulating maintenance rather than initiation of XCI. Furthermore, Firre knockdown did not affect XCI onset in female ES cells.

\section{Conclusions}

In summary, we show that Firre, a lncRNA transcribed from a gene that escapes XCI contributes to the maintenance of a silenced $\mathrm{X}$ chromosome compartment by stabilizing one of the main repressive histone marks on the $\mathrm{Xi}$. Specific CTCF/cohesin binding to the Firre locus on the $\mathrm{Xi}$ and/or the lncRNA itself may play a role in positioning the $\mathrm{Xi}$ near the nucleolus surface and in maintaining one of its epigenetic features. Our results are consistent with XCI being insured by the synergy of multiple layers of control.

\section{Methods}

\section{Mouse embryos, tissues, and cell lines}

Embryos collected from pregnant C57BL/6J (BL6) mice at $12.5 \mathrm{dpc}$ were sexed using PCR primers for the malespecific gene, Sry. Male and female BL6 adult livers were collected. The Patski fibroblast line in which the $\mathrm{Xi}$ is from BL6 and the Xa from M. spretus was originally derived from embryonic kidney $[17,46]$. Female ES cells (PGK12.1) [72], and male ES cells (WD44) were cultured in standard ES medium with 1,000 U/mL leukemia inhibitory factor (LIF) (Millipore) on MEF feeders, and harvested or replated for other experiments after depletion of MEF feeders. Mouse ES cells were differentiated using the standard embryoid body (EB) protocol. The presence of normal X chromosomes was verified by karyotyping. Brains were collected from female F1 obtained by mating M. spretus males (Jackson Labs) with females that carry an Xist mutation (B6.Cg-Xist $<\operatorname{tm} 5$ Sado $>$ ) [73]. F1 mice carrying the mutant Xist allele fail to silence the BL6 $\mathrm{X}$ and thus have completed skewing of inactivation of the spretus X. Primary fibroblasts cultures were derived from ears of male and female BL6 mice. Primary neuron cultures were established from the hippocampus dissected from 0 to 2-day-old mouse pups.

\section{ChIP-chip}

Chromatin immunoprecipitations [74] were performed using $10 \mu \mathrm{g}$ of CTCF antibody (Millipore), $10 \mathrm{ug}$ of RAD21 antibody (Abcam), or $5 \mu \mathrm{g}$ of H3K27me3 antibody (Millipore) as previously described [17]. ChIP-chip was done using Nimblegen genome $385 \mathrm{~K}$ and $2.1 \mathrm{M}$ tiling arrays to cover the entire X (100 bp tiling interval). H3K27me3 ChIP-chip before and after transient knockdown of either Firre or Ctcf in Patski cells were done using Nimblegen 2.1 M promoter arrays (100 bp interval). H3K27me3 ChIP-chip before and after stable knockdown of Firre (siRNA and shRNA) in Patski cells were done using Agilent $1 \mathrm{M}$ mouse promoter arrays (200 bp interval). GFF ratios ( $\log _{2} \mathrm{ChIP} /$ input) and peak files were generated using Nimblescan or Agilent DNA Feature extraction software. Peaks were identified using a 500 bp sliding window with default values from Nimblescan. Metagene profiles were obtained by calculating averages of H3K27me3 enrichment at the $5^{\prime}$ end of genes using a $500 \mathrm{bp}$ sliding window (100 bp interval) for NimbleGen arrays, and a $600 \mathrm{bp}$ sliding window (200 bp interval) for Agilent arrays by end-analysis [75]. Only unique RefSeq genes were included in the analysis. H3K27me3 enrichment at the gene body ( 1 to $2 \mathrm{~kb}$ downstream of TSS) of approximately $600 \mathrm{X}$-linked and approximately 16,000 autosomal genes was compared for each gene to generate scatter plots (Figure 6E, F). Verification of CTCF binding sites by gel mobility shift assays was done as described [76]. 


\section{Allele-specific ChIP-seq}

Allele-specific ChIP-seq was done as described [47]. Four or five CTCF ChIP samples were pooled (approximately 200 to $300 \mathrm{ng}$ ) for library preparation. The library with inserts of 200 to $600 \mathrm{bp}$ was sequenced on Illumina Genome Analyzer, yielding 36 nt single-end reads for ChIP-seq in Patski cells or 100 nt pair-end reads for ChIP-seq in brain. A total of $49.3 \mathrm{M}$ and $283.7 \mathrm{M}$ uniquely mapped reads were obtained for Patski cells and brain, respectively. A pseudo-spretus genome was assembled by substituting available SNPs between BL6 and $M$. spretus into the BL6 reference genome ( $\mathrm{mm} 9)$. SNPs were obtained from the Sanger Institute (SNP database Nov/2011 version) and from our previous study (169,031 additional SNPs, of which 3,571 were X-linked) [17]. Reads were aligned separately to the BL6 reference genome and to the pseudo-spretus genome using BWA/ v0.5.9 with default parameters. Reads that mapped uniquely and with high-quality mapping score (MAPQ $\geq 30$ ) to either the BL6 genome or the pseudo-spretus genome were segregated into three categories: (1) BL6-SNP reads containing only BL6-specific SNPs; (2) spretus-SNP reads containing only spretus-specific SNPs; (3) ambiguous reads that do not contain SNPs or contain both BL6specific SNPs and spretus-specific SNPs. The number of total mapped reads and allele-specific reads in each 100 bp window was calculated and viewed in the UCSC genome browser (Figure 2A). For PolII-S5p ChIP-seq the same allelic analysis was done using $100 \mathrm{nt}$ single-end reads obtained by Hi-seq in Patski cells. In addition, we re-analyzed PolII-S5p ChIP Hi-seq datasets we previously obtained in mouse brain (GSE30761) [47]. PolII-S5p allelic profiles were viewed in the UCSC genome browser (Figure 2B).

\section{Immunofluorescence, RNA-FISH, and DNA-FISH}

Cells cultured on chamber slides or, in the case of neuronal cells, on poly-Lysine pre-coated coverslips for 6day cultures were permeabilized in $0.5 \%$ triton X-100 for $10 \mathrm{~min}$ and fixed with $4 \%$ paraformaldehyde for $10 \mathrm{~min}$. Fixed cells were used for RNA-FISH or DNA-FISH directly using standard protocols. For immunofluorescence (IF), fixed cells were blocked in BSA buffer (4XSSC, $0.42 \% \mathrm{BSA}$ ) for $30 \mathrm{~min}$. After incubation with a primary antibody for nucleophosmin (Abcam) or for H3K27me3 (Millipore) at 1:500 dilution overnight at $4^{\circ} \mathrm{C}$ cells were washed with PBST and incubated with the secondary antibody conjugated to Texas Red or AMCA (Vectashield) at $37^{\circ} \mathrm{C}$ for $1 \mathrm{~h}$. DNA-FISH was done using BAC probes (RP23-338M16 or RP24-322N20 for Firre and RP23299L1 for Dxz4), and RNA-FISH using an Xist plasmid or Firre BAC (RP24-322N20). Probes were labeled with SpectrumGreen or SpectrumRed dUTP (Vysis). For IF combined with RNA-FISH, cells after IF were hybridized with probes overnight at $37^{\circ} \mathrm{C}$ and washed in $50 \%$ formamide $/ 2 \times \mathrm{SSC}$ and $2 \times \mathrm{SSC}$ at $42^{\circ} \mathrm{C}$, prior to Hoechst 33342 (Molecular probes) counterstaining. For IF combined with DNA-FISH, cells after IF were post-fixed in 4\% paraformaldehyde for $10 \mathrm{~min}$, denatured in $70 \%$ formamide $/ 2 \times \mathrm{SSC}$ at $85^{\circ} \mathrm{C}$ for $15 \mathrm{~min}$, dehydrated for $2 \mathrm{~min}$ each in $70 \%, 85 \%$, and $100 \%$ ethanol, prior to overnight hybridization at $42^{\circ} \mathrm{C}$ and washing as described [77]. The association frequency between Firre (or Dxz4) and the nucleolus was scored using a Deltavision deconvolution microscope (Keck Center, University of Washington). At least 100 nuclei were examined in each condition.

\section{Ctcf and Firre RNAi knockdown}

A pool of two 'Silencer select' siRNA duplexes for Firre knockdown (duplex1: sense, 5'-CAGGUACCGUGAG CAAUCAtt-3', antisense, 5'-UGAUUGCUCACGGUAC CUGgt-3', duplex2: sense, 5'-CCUUCAGAGUAUUAA UGCAtt-3', antisense, 5'-UGCAUUAAUACUCUGAAG Ggt-3') and a 'Stealth' 25-mer siRNA duplex for Ctcf knockdown (sense, 5'-UGGACCAGCACAGUUAUCUG CAUGU-3', antisense, 5'-ACAUGCAGAUAACUGUG CUGGUCCA-3') were obtained with a control Stealth oligomer with no specificity to the mouse genome (Invitrogen). Double siRNA transfections were performed using $5 \mu \mathrm{L}$ of lipofectamine mixed with $250 \mu \mathrm{L}$ of Opti$\mathrm{MEM}^{\circ}$ I Reduced Serum Medium containing $100 \mathrm{pmol}$ of each RNAi oligomer. After 20 min incubation the mix was added to $2 \times 10^{5}$ cells in $1.25 \mathrm{~mL}$ DMEM/5\%FBS with no antibiotics. The medium was changed with DMEM/10\% FBS after incubation at $37^{\circ} \mathrm{C}$ for 16 to $24 \mathrm{~h}$. Two days after the first transfection, cells were split and $2 \times 10^{5}$ cells transfected again. Three days later cells were harvested and immunostaining, western blots, and qRT-PCR performed to verify knockdown efficiency. Stable knockdown using Firre shRNA that hit five regions exclusively in the Firre gene (Additional file 1: Figure S1) was done using Addgene's pLKO.1 protocol [78]. Forward oligo, 5' CCGG-CCTTCAGAGTATTAATGCAGA (sense)-CTCG AG-TCTGCATTAATACTCTGAAGG (antisense)-TTTT TG 3', and reverse oligo, 5' AATTCAAAAA-CCTT CAGAGTATTAATGCAGA (sense)-CTCGAG-TCTGCA TTAATACTCTGAAGG (antisense) 3' (IDT) were annealed for cloning into the pLKO.1 vector, as well as control oligos with scramble sequence (Addgene) to generate the control vector. The two plasmids were then used to produce lentiviral particles (Fred Hutchinson Cancer Research Center/CCEH shared resources) for infection of Patski cells and puromycin selection for cells stably expressing shRNA. The knockdown efficiency was determined by qRT-PCR. In the experiments to confirm the effect of Firre depletion of H3K27me3 by ChIP-chip and to examine gene expression changes, control and stable knockdown Patski cells were subject to an additional 
transfection with control and Firre siRNA, respectively, to achieve maximum depletion. Mouse female ES cells (PGK12.1) with control and stable Firre knockdown were established using the same lentiviral particles.

\section{RNA-seq}

RNA-seq indexed libraries were prepared using Illumina TruSeq RNA sample preparation kit for two biological replicates from control or stable knockdown (si\&shRNA) Patski cells, respectively. Libraries from two replicates were pooled for one lane of $36 \mathrm{nt}$ single-end plus index sequencing as described [17]. Reads were mapped and RPKM obtained using TopHat/Cufflinks [79]. A total of 22.3 $\mathrm{M}$ and 23.7 $\mathrm{M}$ mapped reads were obtained from two controls $(11.8 \mathrm{M}$ and $10.5 \mathrm{M})$ and two Firre stable knockdown (11.9 M and $11.7 \mathrm{M})$ replicates, respectively. Differential gene expression was determined by Cuffdiff2 [80]. Functional analysis of upregulated and downregulated genes was done by GO DAVID [52]. Allele-specific RNA-seq analysis was performed as described above. Reads were aligned separately to the BL6 reference genome and transcriptome ( $\mathrm{mm} 9)$ and to the pseudo-spretus genome and transcriptome using Tophat/v2.0.0 with default parameters. Only those reads that mapped uniquely and with a high-quality mapping score (MAPQ $\geq 30$ ) to either the BL6 genome or the pseudo-spretus genome were kept for allele-specific analyses. SNP-read counts from exons of BL6 and spretus alleles were calculated and adjusted by the ratio of the total number of mapped reads between control and knockdown (that is, the SNP-read counts from the knockdown were divided by 1.06 (23.7 M/ $22.3 \mathrm{M}$ ). Since Eif2s3x exons (except exon 1) have a high sequence similarity to another X region (chrX:3168078031684279), we included reads contained in exons with a low MAPQ score for this gene. For Firre we included SNP reads from both exonic and intronic regions.

\section{Quantitative RT-PCR and allele-specific restriction enzyme digestion}

RNA extracted from either mouse tissues or cells by the Qiagen RNeasy kit with on-column DNaseI digestion was reverse transcribed into first-strand cDNAs using SuperScriptII reverse transcriptase (Invitrogen). qRT-PCR was done using gene-specific primers and an ABI7900 PCR system. The primer sequence information is presented in Additional file 14: Table S8. The primers used for Firre qRT-PCR spanning an intron produce an amplicon targeting most of Firre transcripts (Additional file 1: Figure S1). To measure allele-specific expression cDNA was used for 32-cycle PCR with primers designed around the SNPs. PCR products were split into two aliquots, one serving as undigested control while the other was digested with a restriction endonuclease (SnabI for Atp7a, BglII for Pgk1, and KpnI for Iqsec2) (New England Biolabs). Gel elec- trophoresis was performed to examine whether reactivation happened after Firre or Ctcf knockdown.

\section{Statistical analyses}

Two-tailed unpaired student t-tests were used to compare the frequency of association of Firre and Dxz4 to the nucleolus surface between females and males, and between Firre or Ctcf knockdown and control Patski cells. Gene expression ratios between Firre or Ctcf knockdown cells and control cells were compared to a ratio of 1 expected if there was no change in expression using one-sample t-tests. Chi-square tests were done to evaluate changes in the frequency of intense H3K27me3 staining spots between Firre or Ctcf knockdown and control Patski cells. Kolmogorov-Smirnov-tests were used to determine the significance of changes in the distribution of H3K27me3 enrichment within 1 to $2 \mathrm{~kb}$ downstream of the TSS of $\mathrm{X}$-linked or autosomal genes (Figure 6C). Significances of gene expression fold changes measured by RNA-seq analysis of two control and two Firre stable knockdown Patski cells were determined by Cuffdiff2 [80].

\section{Ethics statement}

For mice sacrificed, euthanasia was accomplished using two methods (carbon dioxide asphyxiation followed by cervical dislocation) as required by the University of Washington's Office of Animal Welfare. Husbandry and all other procedures were approved by the University of Washington's Office of Animal Welfare (Protocol 2254).

\section{Additional files}

Additional file 1: Figure S1. Location of qRT-PCR amplicon and of shRNA hits in the Firre gene viewed in the UCSC genome browser. The Firre qRT-PCR amplicon spans an intron and targets most transcripts. The shRNAs hit five regions in Firre, two in exons and three in introns. There is no other hit in the mouse genome from Blat search.

Additional file 2: Figure S2. RNA-FISH fails to detect a Firre signal on the $\mathrm{Xi}$ in Patski cells and detects no changes in Xist cloud after Firre knockdown. (A) Examples of RNA-FISH for Firre (red) and Xist (green) to mark the $X_{i}$ in nuclei of Patski cells. The single bright Firre signal that does not overlap with Xist presumably corresponds to the Xa locus (arrow). (B) No change in the size or shape of Xist RNA clouds is detected in Patski cells after Firre knockdown. Examples of Xist (green) RNA FISH in nuclei of Patski cells after Firre knockdown using shRNA.

Additional file 3: Figure S3. RNA FISH fails to detect Firre transcripts on the $\mathrm{Xi}$ in female MEFs and primary neurons. (A) Examples of MEF nuclei subject to RNA-FISH to detect Xist (red) and Firre (green). Note that one or more bright Firre signals are detected but none overlap with the two Xist clouds that mark the two Xi in this MEF line. (B) Same analysis in primary neurons in which only one $\mathrm{Xi}$ is present.

Additional file 4: Table S1. Genes with significant expression changes between control and Firre knockdown Patski cells.

Additional file 5: Figure S4. Examples of nuclei from fibroblasts that demonstrate the presence of association (A, D) or absence of association $(B, C, E)$ between Firre and Dxz4 $(A, B, C)$, or Dxz4 and Xist $(D, E)$, with the nucleolus (see also Figure 4). (A, B) Nuclei from female ear fibroblasts subjected to DNA-FISH to detect Firre (red), Dxz4 (green), and immunostaining to detect nucleophosmin (blue). (C) Same analysis in male ear fibroblasts. 
(D, E) Nuclei from female Patski cells subjected to DNA-FISH to detect Dxz4 (red), RNA-FISH to detect Xist (green) and mark the Xi, and immunostaining to detect nucleophosmin (blue). One deconvoluted $Z$ section is shown.

Additional file 6: Figure S5. Efficiency of Ctcf knockdown and H3K27me3 changes after Firre stable knockdown. (A) qRT-PCR (left) and western blots (right) confirm Ctcf knockdown in Patski cells using siRNA Error bars indicate s.e.m. (B) H3K27me3 enrichment is reduced at the $5^{\prime}$ end of $X$-linked genes $(X)$ but not autosomal genes $(A)$ after Firre stable knockdown in Patski cells using shRNA and siRNA. Metagene analysis shows average H3K27me3 enrichment (log2 ChIP/input) 3 kb upstream and downstream of the transcription start site (TSS) for 647 X-linked versus 16,141 autosomal genes. See also Figure $6 B$. Note the reduction in H3K27me3 at the TSS in Figure 6B is probably due to artifacts from hybridization or types of promoter array used, which is not observed here.

Additional file 7: Table S2. H3K27me3 enrichment at 10 escape genes in control and Firre knockdown Patski cells.

Additional file 8: Table S3. Functional analysis of genes significantly misregulated upon Firre knockdown in Patski cells.

Additional file 9: Table S4. Expression changes for genes involved in heterochromatin and gene silencing between Firre knockdown and control Patski cells.

Additional file 10: Table S5. Number of alleles with expression changes between control and Firre knockdown Patski cells.

Additional file 11: Table S6. Expression changes for genes with $\geq 5$ SRPM on the Xi after Firre knockdown in Patski cells.

Additional file 12: Table S7. Expression changes for escape genes after Firre knockdown in Patski cells.

Additional file 13: Figure S6. Firre stable knockdown in female mouse ES cells did not disrupt Xist upregulation/coating during differentiation.

(A) Evidence of stable Firre knockdown (KD) in female mouse ES cells PGK12.1 using shRNA. QRT-PCR was performed at day 3 and day 10 after infection with lentiviral particles carrying control scramble shRNA or Firre shRNA. Puromycin was added 1 day after infection to select cells expressing shRNA. (B) Similar Xist expression fold change are observed in undifferentiated and differentiated PGK12.1 ES cells (see also Figure 7G) and in Patski cells (see also Figure 7E) upon Firre knockdown. (C) Xist cloud shape and size is similar between control and knockdown PGK12.1 cells at day 14. Examples of Xist (green) RNA-FISH in three nuclei for each condition are shown.

Additional file 14: Table S8. Sequences of primers.

\section{Competing interests}

The authors declare that they have no competing interests.

\section{Authors' contributions}

FY discovered the CTCF binding clusters specific to the inactive $X$ chromosome and demonstrated association to the nucleolus. FY and XD characterized CTCF and RAD21 binding at Firre in ES cells, embryos and tissues, as well as performed the knockdown experiments. JBB provided the mouse brain used for allele-specific studies. GW and JX provided primary neuron cultures. JS obtained the DNA sequences for RNA-seq and ChIP-seq. YL obtained ChIP-chip data using Agilent arrays. NR, JMM, and GNF helped with the Ctcf knockdown. WM and WSN did the bioinformatic analyses of sequence. $F Y, X D$, and WM did statistical analyses of the data. FY, XD, and CMD wrote the manuscript. All authors read and approved the final manuscript.

\section{Acknowledgments}

$F Y, X D, J B B$, and CMD are supported by grant GM046883 from the National Institutes of Health (NIH.gov). XD, CMD, GW, and JX are also supported by grant MH083949 from the $\mathrm{NIH}$. WM and WSN are supported by grant GM098039 from the $\mathrm{NIH}$. XD is also supported by a Junior Faculty Pilot award from the Department of Pathology at the University of Washington. We thank N. Brockdorff (Oxford University) for the female ES cells (PGK12.1), C. Ware (University of Washington) for the male ES cells (WD44), and T. Sado (Kyushu University) for the Xist mutant mice (B6.Cg-Xist < tm5Sado>). We thank S. and J. Henikoff (Fred Hutchinson Cancer Research Center, Seattle) for access to their computational analysis platform, and M. Andronescu for her help with data analyses. We thank X. Chen and Y. Zhou (University of Washington) for their help with imaging and CGH array analyses.

\section{Accession numbers}

The RNA-seq, ChIP-chip and ChIP-seq data are available in the Gene Expression Omnibus (GEO) database, under the accession number GSE30761 and GSE59779.

\section{Author details}

${ }^{1}$ Department of Pathology, University of Washington, Seattle, Washington 98195, USA. ²Department of Genome Sciences, University of Washington, Seattle, Washington 98195, USA. ${ }^{3}$ Division of Human Biology, Fred Hutchinson Cancer Research Center, Seattle, Washington 98109, USA. ${ }^{4}$ Department of Integrative Physiology and Neuroscience, Washington State University, Pullman, Washington 99164, USA. ${ }^{5}$ Department of Computer Science and Engineering, University of Washington, Seattle, Washington 98155, USA. ${ }^{6}$ Department of Medicine, University of Washington, Seattle, Washington 98195, USA.

Received: 16 July 2014 Accepted: 23 February 2015

Published online: 12 March 2015

\section{References}

1. Lyon M. Gene action in the X-chromosome of the mouse (Mus musculus L). Nature. 1961;190:372-3.

2. Lessing $D$, Lee JT. $X$ chromosome inactivation and epigenetic responses to cellular reprogramming. Annu Rev Genomics Hum Genet. 2013;14:85-110.

3. Schulz EG, Heard E. Role and control of X chromosome dosage in mammalian development. Curr Opin Genet Dev. 2013;23:109-15.

4. Engreitz JM, Pandya-Jones A, McDonel P, Shishkin A, Sirokman K, Surka C, et al. The Xist IncRNA exploits three-dimensional genome architecture to spread across the X chromosome. Science. 2013;341:1237973.

5. Simon MD, Pinter SF, Fang R, Sarma K, Rutenberg-Schoenberg M, Bowman SK, et al. High-resolution Xist binding maps reveal two-step spreading during X-chromosome inactivation. Nature. 2013;504:465-9.

6. Heard $\mathrm{E}$, Disteche $\mathrm{CM}$. Dosage compensation in mammals: fine-tuning the expression of the X chromosome. Genes Dev. 2006;20:1848-67.

7. Gartler SM, Riggs AD. Mammalian X-chromosome inactivation. Annu Rev Genet. 1983;17:155-90.

8. Gendrel AV, Apedaile A, Coker H, Termanis A, Zvetkova I, Godwin J, et al. Smchd1-dependent and -independent pathways determine developmental dynamics of CpG island methylation on the inactive $x$ chromosome. Dev Cell. 2012;23:265-79.

9. Brown CJ, Willard HF. The human X-inactivation centre is not required for maintenance of X-chromosome inactivation. Nature. 1994;368:154-6.

10. Yildirim E, Kirby JE, Brown DE, Mercier FE, Sadreyev RI, Scadden DT, et al. Xist RNA is a potent suppressor of hematologic cancer in mice. Cell. 2013;152:727-42.

11. Csankovszki G, Nagy A, Jaenisch R. Synergism of Xist RNA, DNA methylation, and histone hypoacetylation in maintaining $X$ chromosome inactivation. J Cell Biol. 2001;153:773-84.

12. Escamilla-Del-Arenal M, da Rocha ST, Heard E. Evolutionary diversity and developmental regulation of X-chromosome inactivation. Hum Genet. 2011;130:307-27.

13. Escamilla-Del-Arenal M, da Rocha ST, Spruijt CG, Masui O, Renaud O, Smits $\mathrm{AH}$, et al. Cdyl, a new partner of the inactive $\mathrm{X}$ chromosome and potential reader of H3K27me3 and H3K9me2. Mol Cell Biol. 2013;33:5005-20.

14. Gendrel AV, Heard E. Noncoding RNAs and epigenetic mechanisms during X-chromosome inactivation. Annu Rev Cell Dev Biol. 2014;30:561-80.

15. Kalantry S, Mills KC, Yee D, Otte AP, Panning B, Magnuson T. The Polycomb group protein Eed protects the inactive $X$-chromosome from differentiation-induced reactivation. Nat Cell Biol. 2006;8:195-202.

16. Berletch JB, Yang F, Xu J, Carrel L, Disteche CM. Genes that escape from X inactivation. Hum Genet. 2011;130:237-45.

17. Yang F, Babak T, Shendure J, Disteche CM. Global survey of escape from X inactivation by RNA-sequencing in mouse. Genome Res. 2010;20:614-22.

18. Calabrese JM, Sun W, Song L, Mugford JW, Williams L, Yee D, et al. Site-specific silencing of regulatory elements as a mechanism of $X$ inactivation. Cell. 2012;151:951-63.

19. Finn EH, Smith $\mathrm{CL}$, Rodriguez J, Sidow A, Baker JC. Maternal bias and escape from $X$ chromosome imprinting in the midgestation mouse placenta. Dev Biol. 2014;390:80-92. 
20. Wu H, Luo J, Yu H, Rattner A, Mo A, Wang Y, et al. Cellular resolution maps of $X$ chromosome inactivation: implications for neural development, function, and disease. Neuron. 2014;81:103-19.

21. Hacisuleyman E, Goff LA, Trapnell C, Williams A, Henao-Mejia J, Sun L, et al. Topological organization of multichromosomal regions by the long intergenic noncoding RNA Firre. Nat Struct Mol Biol. 2014;21:198-206.

22. Chow JC, Heard E. Nuclear organization and dosage compensation. Cold Spring Harb Perspect Biol. 2010;2:a000604.

23. Masui O, Bonnet I, Le Baccon P, Brito I, Pollex T, Murphy N, et al. Live-cell chromosome dynamics and outcome of $X$ chromosome pairing events during ES cell differentiation. Cell. 2011;145:447-58.

24. Augui S, Nora EP, Heard E. Regulation of X-chromosome inactivation by the X-inactivation centre. Nat Rev Genet. 2011;12:429-42.

25. Barr ML, Bertram EG. A morphological distinction between neurones of the male and female, and the behaviour of the nucleolar satellite during accelerated nucleoprotein synthesis. Nature. 1949;163:676

26. Chadwick BP, Willard HF. Chromatin of the Barr body: histone and non-histone proteins associated with or excluded from the inactive $X$ chromosome. Hum Mol Genet. 2003;12:2167-78.

27. Zhang LF, Huynh KD, Lee JT. Perinucleolar targeting of the inactive $X$ during $S$ phase: evidence for a role in the maintenance of silencing. Cell. 2007;129:693-706.

28. Filippova GN. Genetics and epigenetics of the multifunctional protein CTCF. Curr Top Dev Biol. 2008:80:337-60.

29. Phillips JE, Corces VG. CTCF: master weaver of the genome. Cell. 2009;137:1194-211.

30. Ong CT, Corces VG. CTCF: an architectural protein bridging genome topology and function. Nat Rev Genet. 2014;15:234-46.

31. Nakahashi H, Kwon KR, Resch W, Vian L, Dose M, Stavreva D, et al. A genome-wide map of CTCF multivalency redefines the CTCF code. Cell Rep. 2013;3:1678-89.

32. Merkenschlager M, Odom DT. CTCF and cohesin: linking gene regulatory elements with their targets. Cell. 2013;152:1285-97.

33. Yusufzai TM, Tagami H, Nakatani Y, Felsenfeld G. CTCF tethers an insulator to subnuclear sites, suggesting shared insulator mechanisms across species. Mol Cell. 2004;13:291-8.

34. Yusufzai TM, Felsenfeld G. The $5^{\prime}$-HS4 chicken \{beta\}-globin insulator is a CTCF-dependent nuclear matrix-associated element. Proc Natl Acad Sci U S A. 2004;101:8620-4.

35. Ottaviani A, Schluth-Bolard C, Rival-Gervier S, Boussouar A, Rondier D, Foerster AM, et al. Identification of a perinuclear positioning element in human subtelomeres that requires A-type lamins and CTCF. EMBO J. 2009:28:2428-36.

36. Carretero M, Remeseiro S, Losada A. Cohesin ties up the genome. Curr Opin Cell Biol. 2010;22:781-7.

37. Filippova GN, Cheng MK, Moore JM, Truong JP, Hu YJ, Nguyen DK, et al. Boundaries between chromosomal domains of $X$ inactivation and escape bind CTCF and lack CpG methylation during early development. Dev Cell. 2005:8:31-42.

38. Chadwick BP. DXZ4 chromatin adopts an opposing conformation to that of the surrounding chromosome and acquires a novel inactive $X$-specific role involving CTCF and antisense transcripts. Genome Res. 2008;18:1259-69.

39. Horakova AH, Moseley SC, McLaughlin CR, Tremblay DC, Chadwick BP. THE macrosatellite dxz4 mediates ctcf-dependent long-range intrachromosomal interactions on the human inactive X chromosome. Hum Mol Genet. 2012;21:4367-77.

40. Rao SS, Huntley MH, Durand NC, Stamenova EK, Bochkov ID, Robinson JT, et al. A 3D Map of the human genome at kilobase resolution reveals principles of chromatin looping. Cell. 2014;159:1665-80.

41. Parelho V, Hadjur S, Spivakov M, Leleu M, Sauer S, Gregson HC, et al. Cohesins functionally associate with CTCF on mammalian Chromosome Arms. Cell. 2008;132:422-33.

42. Wendt KS, Yoshida K, Itoh T, Bando M, Koch B, Schirghuber E, et al. Cohesin mediates transcriptional insulation by CCCTC-binding factor. Nature. 2008:451:796-801.

43. Stedman W, Kang H, Lin S, Kissil JL, Bartolomei MS, Lieberman PM. Cohesins localize with CTCF at the KSHV latency control region and at cellular c-myc and H19/lgf2 insulators. EMBO J. 2008;27:654-66.

44. Rubio ED, Reiss DJ, Welcsh PL, Disteche CM, Filippova GN, Baliga NS, et al. CTCF physically links cohesin to chromatin. Proc Natl Acad Sci U S A. 2008;105:8309-14.

45. Bernstein BE, Birney E, Dunham I, Green ED, Gunter C, Snyder M. An integrated encyclopedia of DNA elements in the human genome. Nature. 2012:489:57-74
46. Lingenfelter PA, Adler DA, Poslinski D, Thomas S, Elliott RW, Chapman VM, et al. Escape from $X$ inactivation of Smcx is preceded by silencing during mouse development. Nat Genet. 1998;18:212-3.

47. Deng X, Berletch JB, Ma W, Nguyen DK, Hiatt JB, Noble WS, et al. Mammalian $X$ upregulation is associated with enhanced transcription initiation, RNA half-life, and MOF-mediated H4K16 acetylation. Dev Cell. 2013;25:55-68

48. Grant CE, Bailey TL, Noble WS. FIMO: scanning for occurrences of a given motif. Bioinformatics. 2011;27:1017-8.

49. Kung JT, Kesner B, An JY, Ahn JY, Cifuentes-Rojas C, Colognori D, et al. Locus-specific targeting to the $X$ chromosome revealed by the RNA interactome of CTCF. Mol Cell. 2015;57:361-75.

50. Horakova AH, Calabrese JM, McLaughlin CR, Tremblay DC, Magnuson T, Chadwick BP. The mouse DXZ4 homolog retains Ctcf binding and proximity to Pls3 despite substantial organizational differences compared to the primate macrosatellite. Genome Biol. 2012;13:R70

51. Pohlers M, Calabrese JM, Magnuson T. Small RNA expression from the human macrosatellite DXZ4. G3 (Bethesda). 2014;4:1981-9.

52. da Huang W, Sherman BT, Lempicki RA. Systematic and integrative analysis of large gene lists using DAVID bioinformatics resources. Nat Protoc. 2009:4:44-57.

53. Payer B, Lee JT. X chromosome dosage compensation: how mammals keep the balance. Annu Rev Genet. 2008;42:733-72

54. Donohoe ME, Silva SS, Pinter SF, Xu N, Lee JT. The pluripotency factor Oct4 interacts with Ctcf and also controls X-chromosome pairing and counting. Nature. 2009;460:128-32.

55. Hasegawa Y, Brockdorff N, Kawano S, Tsutui K, Tsutui K, Nakagawa S. The matrix protein hnRNP $U$ is required for chromosomal localization of Xist RNA. Dev Cell. 2010;19:469-76.

56. Keohane AM, O'Neill LP, Belyaev ND, Lavender JS, Turner BM. X-Inactivation and histone $\mathrm{H} 4$ acetylation in embryonic stem cells. Dev Biol. 1996;180:618-30.

57. Padeken J, Heun P. Nucleolus and nuclear periphery: velcro for heterochromatin. Curr Opin Cell Biol. 2014;28:54-60.

58. Mahadevaiah SK, Royo H, VandeBerg JL, McCarrey JR, Mackay S, Turner JM. Key features of the $\mathrm{X}$ inactivation process are conserved between marsupials and eutherians. Curr Biol. 2009;19:1478-84.

59. Grant J, Mahadevaiah SK, Khil P, Sangrithi MN, Royo H, Duckworth J, et al. Rsx is a metatherian RNA with Xist-like properties in X-chromosome inactivation. Nature. 2012;487:254-8.

60. MCNairn AJ, Gerton JL. The chromosome glue gets a little stickier. Trends Genet. 2008;24:382-9.

61. Padeken J, Mendiburo MJ, Chlamydas S, Schwarz HJ, Kremmer E, Heun P. The nucleoplasmin homolog NLP mediates centromere clustering and anchoring to the nucleolus. Mol Cell. 2013;50:236-49.

62. Xie W, Barr CL, Kim A, Yue F, Lee AY, Eubanks J, et al. Base-resolution analyses of sequence and parent-of-origin dependent DNA methylation in the mouse genome. Cell. 2012;148:816-31.

63. Hark AT, Schoenherr CJ, Katz DJ, Ingram RS, Levorse JM, Tilghman SM. CTCF mediates methylation-sensitive enhancer-blocking activity at the H19/lgf2 locus. Nature. 2000:405:486-9.

64. Bell AC, West AG, Felsenfeld G. The protein CTCF is required for the enhancer blocking activity of vertebrate insulators. Cell. 1999;98:387-96.

65. Giacalone J, Friedes J, Francke U. A novel GC-rich human macrosatellite VNTR in Xq24 is differentially methylated on active and inactive X chromosomes. Nat Genet. 1992;1:137-43.

66. Keller C, Kulasegaran-Shylini R, Shimada Y, Hotz HR, Buhler M. Noncoding RNAs prevent spreading of a repressive histone mark. Nat Struct Mol Biol. 2013;20:994-1000.

67. Pandey RR, Mondal T, Mohammad F, Enroth S, Redrup L, Komorowski J, et al. Kcnq1ot1 antisense noncoding RNA mediates lineage-specific transcriptional silencing through chromatin-level regulation. Mol Cell. 2008;32:232-46.

68. Fedoriw AM, Calabrese JM, Mu W, Yee D, Magnuson T. Differentiation-driven nucleolar association of the mouse imprinted Kcnq1 locus. G3 (Bethesda) 2012;2:1521-8

69. Csankovszki G, Panning B, Bates B, Pehrson JR, Jaenisch R. Conditional deletion of Xist disrupts histone macroH2A localization but not maintenance of X inactivation. Nat Genet. 1999;22:323-4.

70. Ohhata T, Wutz A. Reactivation of the inactive $X$ chromosome in development and reprogramming. Cell Mol Life Sci. 2012;70:2443-61. 
71. Hansen RS, Gartler SM. 5-Azacytidine-induced reactivation of the human $X$ chromosome-linked $P G K 1$ gene is associated with a large region of cytosine demethylation in the 5' CpG island. Proc Natl Acad Sci U S A. 1990;87:4174-8.

72. Penny GD, Kay GF, Sheardown SA, Rastan S, Brockdorff N. Requirement for Xist in $X$ chromosome inactivation. Nature. 1996;379:131-7.

73. Hoki Y, Kimura N, Kanbayashi M, Amakawa Y, Ohhata T, Sasaki H, et al. A proximal conserved repeat in the Xist gene is essential as a genomic element for X-inactivation in mouse. Development. 2009;136:139-46.

74. Otto SP, Pannell JR, Peichel CL, Ashman TL, Charlesworth D, Chippindale AK, et al. About PAR: the distinct evolutionary dynamics of the pseudoautosomal region. Trends Genet. 2011;27:358-67.

75. Mito Y, Henikoff JG, Henikoff S. Genome-scale profiling of histone H3.3 replacement patterns. Nat Genet. 2005;37:1090-7.

76. Filippova GN, Thienes CP, Penn BH, Cho DH, Hu YJ, Moore JM, et al. CTCF-binding sites flank $C T G / C A G$ repeats and form a methylation- sensitive insulator at the DM1 locus. Nat Genet. 2001;28:335-43.

77. Chadwick BP, Willard HF. Histone H2A variants and the inactive $X$ chromosome: identification of a second macroH2A variant. Hum Mol Genet. 2001;10:1101-13.

78. Moffat J, Grueneberg DA, Yang X, Kim SY, Kloepfer AM, Hinkle G, et al. A lentiviral RNAi library for human and mouse genes applied to an arrayed viral high-content screen. Cell. 2006;124:1283-98.

79. Trapnell C, Pachter L, Salzberg SL. TopHat: discovering splice junctions with RNA-Seq. Bioinformatics. 2009;25:1105-11.

80. Trapnell C, Hendrickson DG, Sauvageau M, Goff L, Rinn JL, Pachter L. Differential analysis of gene regulation at transcript resolution with RNA-seq. Nat Biotechnol. 2013;31:46-53.

\section{Submit your next manuscript to BioMed Central and take full advantage of:}

- Convenient online submission

- Thorough peer review

- No space constraints or color figure charges

- Immediate publication on acceptance

- Inclusion in PubMed, CAS, Scopus and Google Scholar

- Research which is freely available for redistribution 